\title{
Structured Compute-and-Forward with Phase Precoding Scheme: Beyond Integers
}

\author{
Ehsan E. Khaleghi \\ Communications \& Electronics Department \\ TELECOM ParisTech \\ Paris, France \\ E-mail: ehsan.ebrahimi-khaleghi@telecom-paristech.fr
}

\author{
Jean-Claude Belfiore \\ Communications \& Electronics Department \\ TELECOM ParisTech \\ Paris, France \\ E-mail: jean-claude.belfiore@telecom-paristech.fr
}

\begin{abstract}
In this work, we focus on the $K$-user Gaussian Symmetric Complex-valued Interference Channels (GS-CIC). The Compute-and-Forward (CoF) protocol in wireless networks have been employed for Gaussian Symmetric Real-valued Interference Channels (GS-RIC) to achieve approximately the capacity of such channels and making Interference Alignment (IA) by solving a system of linear equations at destinations. We aim to adapt this protocol to GS-CIC. At high Signal-to-Noise Ratio (SNR), this protocol shows a fading behavior of the achievable sum-rate for strong and very strong interference regimes. In complex field this random-like behavior is related to the phase of complex cross channel coefficients. To avoid this fading-like behavior, we consider $\mathbb{Z}[i]$-lattice codes and we propose a phase precoding scheme for CoF protocol with partial feedback. In this method the phase of channel coefficients will then be fed back to the transmitters in order to choose the best precoder factors to control this random behavior. We further simulate the achievable sum-rate given by this scheme and showing significant gain improvement can be obtained in terms of limiting the random behavior of achievable sum-rate.
\end{abstract}

Index Terms-Compute-and-forward, Hermitian form, lattice codes, phase precoding, feedback.

\section{INTRODUCTION}

Today the connection between different communication systems is realized via wireless communication mediums. The most important benefit of using a wireless medium is to have more reliable communication for users in terms of mobility, but it will have its own disadvantages such as communication coverage, interference between users (or channels), fading behavior of transmission channels and transmission power. Managing the interference is an issue to overcome when users in wireless networks want to have at the same time an acceptable Quality of Service (QoS) and higher transmission rates. Rather than avoiding the interference, it can be used in an intelligent way to recover the desired information for an intended receiver. This interference management technique is called IA. CoF protocol in wireless communication is a novel relaying strategy in which relays can decode or compute the functions of transmitted signals from different transmitters, then forward them to the intended destination. By using this protocol interference could be managed by solving a system of linear equations at destinations and the achievable sum-rate will be higher. It can be said IA is possible via CoF protocol.

\section{A. Related Work}

Interference and its influence in communication channels have been introduced many years ago in [1],[2]. Different alignment approaches are known in literature to manage interference in GS-RIC. Two general categories of IA are known as linear IA [3] and non-linear IA [4]. Making IA in an intelligent way is one of the most important challenges in the domain of multi-user wireless information theory. The well known pioneers of the domain have been focused on the case of 2-user (or 3-user) systems to understand the importance of alignment approaches and its difficulties before generalizing them for the general $K$-user systems when $K>2$. This will led us to understand how much gain we can benefit by using different alignment strategies and coding methods. For $2-$ user case much significant progress had been done for strong [5] and very strong interference [6] channels. In [7], first we have showed our interest to use CoF protocol as an IA technique, then we have studied deeply the main framework of Nazer and Gastpar scheme defined in [8]. We also analyzed the new method of $\mathrm{CoF}$ described in [9] to find the cause of random like-behavior of final achievable sum-rate showed for 2-user GS-RIC. This fading behavior is limited by using Golden ratio, its equivalents and our proposed precoding scheme. The new method of using lattice codes over Eisentsein integers has been defined for CoF by Tunali et al. [10] to show higher information rates than reported in [8] can be achieved. Many research studies and code designs have been done for the case of real-valued interference channels, but for the complexvalued interference channels and its code structures still we need to realize much more research.

\section{B. Summary of Paper Results}

In this paper our principal goal is to apply the computation rate defined in [8] by Nazer and Gastpar for GS-CIC. After defining this computation rate, we will use the new $\mathrm{CoF}$ protocol described in [9] by Ordentlich et al. for transforming approximately the $K$-user GS-CIC to the 2 -user case. This transformation will help us to calculate the achievable sum-rate at destinations. Without using any Channel State Information (CSI) at transmitters the performance showed in Fig. 1 is reachable by using the $\mathrm{CoF}$ protocol described in [9]. For the high values of SNR, we are interested in reducing the 
gap between the upper bound and the achievable sum-rate in strong and very strong interference regimes. We can notice, the small variation of the interfering channel gain could result deep fadings on the achievable sum-rate of our transmission channel and it can dramatically reduce the final sum-rate.

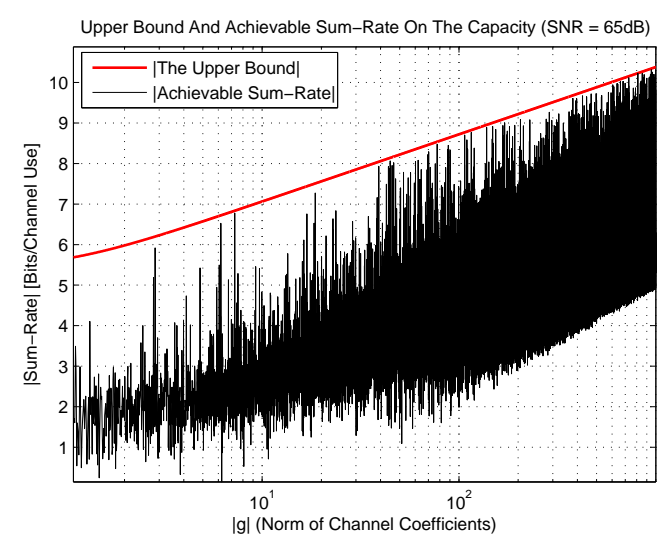

Fig. 1. Upper and lower bounds on the capacity of a 2-user GS-CIC with respect to the cross-gain $g=\rho e^{i \phi} .1<\rho \leq 10^{3}$ and $\phi$ is chosen randomly in $[-\pi / 4,+\pi / 4]$ for each vaiation of $\rho$.

In second part of this work we introduce channel model, lattice structures and the complex case is defined for 2user Gaussian symmetric interference channels (GS-IFC). In third part, the minimum mean square error (MMSE) estimator, the correspondent Hermitian form and the achievable sumrate are defined by using the main framework of [8] and [9]. After showing the benefit of using a phase precoding scheme at transmitters, the new protocol of CoF combined with phase precoders is introduced. The phase precoders will use CSI as a feedback information to calculate the best phase precoder coefficients to improve the final achievable sum-rate. Our proposed precoding scheme also belongs to the general framework introduced by A. Sakzad et al. in [11]; however, this scheme has not been analyzed in detail. Finally, at the last part of this work, the numerical results are presented for high SNR and strong interference channels.

\section{Notational Convention}

Through this paper, we use $\mathbb{C}, \mathbb{Z}$ and $\mathbb{Z}[i]$ to denote the field of complex numbers, the set of integers and Gaussian integers, respectively. $\mathbb{F}_{q}$ to denote the finite field of size $q$. Here in all equations, bold letters are for vectors and bold capital letters are for matrices, e.g., $\mathbf{x}$ and $\mathbf{X}$, respectively. We define $\log _{2}^{+}(x) \triangleq \max \left(\log _{2}(x), 0\right)$. We refer to lattices over complex integers as $\mathbb{Z}[i]$-lattices. We let $|z|$ and $\arg (z)$ denote the modulus and the phase of the complex number $z$, respectively.

\section{CHANNEL MODEL, LATTICE STRUCTURE AND THE COMPLEX CASE}

\section{A. Channel Model}

The $K$-user GS-CIC is the general model of this paper. The transmitters reliably communicate linear functions to $M$ relays over a complex-valued symmetric interference channel. In this model each relay $m$ observes a noisy linear combination of the transmitted signals through the channel,

$$
y_{m}=\sum_{l=1}^{K} h_{m l} \cdot x_{l}+z_{m}
$$

where $h_{m l} \in \mathbb{C}$ are the channel coefficients, $\mathbf{x}=$ $\left[x_{1}, \cdots, x_{K}\right], \mathbf{y}=\left[y_{1}, \cdots, y_{K}\right]$ and $\mathbf{z}=\left[z_{1}, \cdots, z_{K}\right]$ are used to denote the input-vector, the output-vector and the complex i.i.d Gaussian noise-vector all of size $K$, respectively. Furthermore, $\mathbf{z}_{m} \sim \mathcal{C N}\left(0, \sigma^{2}\right)$ and $\mathbf{x}_{l} \in \Lambda_{c}$. Let $\Lambda_{c}$ be the coding lattice which is common to all users. In this paper all users have the same power constraint i.e., $P_{\mathrm{i}}=P$, so the SNR is defined as SNR $=\frac{P}{\sigma^{2}}$. By considering a simple lattice IA defined in [9] this $K$-user case will be approximately equivalent to a 2 -user case showed in Fig. 2, this means that,

$$
\mathbf{H}=\left[\begin{array}{ll}
1 & g \\
g & 1
\end{array}\right]
$$

which the channel coefficients are considered to be in the field of complex numbers.

The simplified expression of (1) for 2 -user case is written as,

$$
\left[\begin{array}{l}
y_{1} \\
y_{2}
\end{array}\right]=\mathbf{H} \cdot\left[\begin{array}{l}
x_{1} \\
x_{2}
\end{array}\right]+\left[\begin{array}{l}
z_{1} \\
z_{2}
\end{array}\right]
$$

What we see in Fig. 2] the channel is symmetric, it means $\mathbf{H}(i, j)=g$ for all $i \neq j$, and $\mathbf{H}(i, i)=1$ for all $i$ 's.

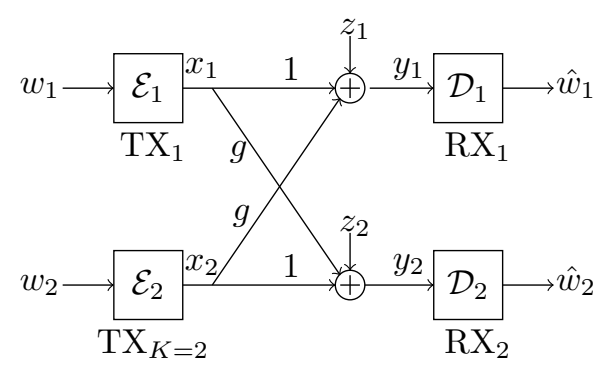

Fig. 2. 2-user Gaussian Symmetric complex-valued Interference Channel.

In addition, each transmitter must satisfy the power constraint, which for $n$ channel uses and user $i$ is given by:

$$
\frac{1}{n} \sum_{j=0}^{n}\left|\mathbf{x}_{i}\right|^{2} \leq P_{i}
$$

In order to perform CoF, we need a couple of ingredients:

- Each transmitter is equipped with an encoder $\mathcal{E}_{l}$, that maps length $-K$ messages over a lattice code.

- Each receiver will recover the transmitted messages by using a decoder $\mathcal{D}_{l}$, that maps the received messages over a lattice code to its transmitted version. This mapping is possible with $K$ integers $\left\{a_{1}, \cdots, a_{K}\right\}$ which will 
approximate the channel gains $h_{m l}$. The main idea is that, if $\mathbf{x}_{l} \in \Lambda_{c}$ and if $\Lambda_{c}$ is a coding complex lattice, then

$$
\lambda \triangleq \sum_{l=1}^{K} a_{l} \mathbf{x}_{l} \in \Lambda_{c}
$$

as well.

\section{B. Lattice structure}

We recall some fundamental notation of lattice codes which are essential throughout the paper. The definition of a $\mathrm{N}$ dimension complex lattice $\Lambda$ with generator matrix $(G) \triangleq$ $\left(\mathbf{g}_{1}^{T}\left|\mathbf{g}_{2}^{T}\right| \ldots \mid \mathbf{g}_{N}^{T}\right)$, for all $\mathbf{g}_{j} \in \mathbb{C}^{m}$ and $1 \leq j \leq N$ is the set of points in $\mathbb{C}^{m}$ (the $\mathbf{g}_{i}^{T}$ vectors are considered to be the columns of $\mathbf{G}$ )

$$
\Lambda=\left\{\mathbf{x}=\mathbf{u G} \mid \mathbf{u} \in \mathbb{Z}[i]^{m}\right\}
$$

when $m=N$, the lattice is called full rank. Around each lattice point $\mathbf{x} \in \Lambda$ there is a Voronoi region $\nu(\mathbf{x})$ defined as:

$$
\nu(\mathbf{x})=\left\{\mathbf{y} \in \mathbb{C}^{m}:\|\mathbf{y}-\mathbf{x}\| \leq\|\mathbf{y}-\lambda\|, \forall \lambda \in \Lambda\right\}
$$

If $\Lambda^{\prime}$ is a lattice itself, then a subset $\Lambda^{\prime} \subseteq \Lambda$ is called a sublattice. By having a sublattice $\Lambda^{\prime}$, the lattice code $\Lambda / \Lambda^{\prime}$ can be define. $\Lambda / \Lambda^{\prime}$ includes a finite constellation of lattice points from the lattice $\Lambda$. An intelligent choice [12] for the sublattice $\Lambda^{\prime}$ is $a \Lambda$ for some $a \in \mathbb{Z}[i]$. The shape of the lattice constellation is determined by the Voronoi region of the lattice $\Lambda^{\prime}$. we can also define the lattice modulo operation as

$$
\begin{gathered}
Q_{\Lambda}(\mathbf{x}) \triangleq \underset{\lambda \in \Lambda}{\operatorname{argmin}}\|\mathbf{y}-\lambda\| \\
\mathbf{y} \bmod \Lambda \triangleq \mathbf{y}-Q_{\Lambda}(\mathbf{x})
\end{gathered}
$$

The generator matrix of a lattice can be found by applying the Cholesky decomposition to a definite positive Hermitian form of a complex lattice.

\section{The Complex Case}

In the complex case, we have another degree of freedom. Now, instead of dealing with $\mathbb{Z}$-lattices, we consider an $\mathcal{O}_{\mathbb{K}}$-lattice $\Lambda_{c}$ where $\mathcal{O}_{\mathbb{K}}$ is the ring of integers of a complex quadratic number field $\mathbb{K}=\mathbb{Q}(\sqrt{-b})$ for some square free positive integers $b$. When $\mathcal{O}_{\mathbb{K}}$ is a Principal Ideal Domain (PID), then the definition of an $\mathcal{O}_{\mathbb{K}}$-lattice is similar to the one of a $\mathbb{Z}$-lattice, and there exists lattice basis. Unfortunately, when $\mathbb{K}$ is a complex quadratic number field, then $\mathcal{O}_{\mathbb{K}}$ is not in general a PID. It is a PID when $b \in\{1,2,3,7,11,19,43,67,167\}$. The most natural choice, in the complex case, is to mimic the real case, that is take $b=1$, which implies $\mathcal{O}_{\mathbb{K}}=\mathbb{Z}[i]$. A more judicious choice has been made in [10] with $b=3$ giving rise to $\mathcal{O}_{\mathbb{K}}=\mathbb{Z}[\omega]$ where $\omega=\zeta_{3} \mathbb{1}$. As a lattice, $\mathbb{Z}[\omega] \simeq A_{2}$, that is the hexagonal lattice, when $\mathbb{Z}[i] \simeq \mathbb{Z}^{2}$. This is explained by the fact that $A_{2}$ is better for quantization than $\mathbb{Z}_{2}$.

\footnotetext{
${ }^{1}$ Here, as usually, $\zeta_{q}$ denotes a primitive $q^{t h}$-root of unity.
}

\section{MMSE ESTIMATOR AND THE}

\section{COMPUTE-AND-FORWARD With PHASE PRECODING}

Suppose that we code over $n$ time-slots where $n$ is the degree of the relative number field $\mathbb{K}$ over quadratic complex field $\mathbb{F}$ which can be, for instance, $\mathbb{Z}[i]$ or $\mathbb{Z}[\omega]$. In this part first we aim at computing the MMSE, $n \times n$ matrix $\mathrm{B}$, Then we define our proposed phase precoding scheme to improve the behavior of the achievable sum-rate.

\section{A. the minimum mean square error estimator}

We suppose, for sake of simplicity, that all transmitted signals $\mathbf{x}_{k}$ are coming from a 1-dimensional $\mathcal{O}_{\mathbb{K}}$-lattice, which is obviously $\mathcal{O}_{\mathbb{K}}$ itself. Let $\operatorname{Gal}_{\mathbb{K} / \mathbb{F}}=\left\{\sigma_{0}, \sigma_{1} \ldots, \sigma_{n-1}\right\}$ denotes the Galois group of the extension $\mathbb{K}$ over $\mathbb{F}$ and set

$$
\mathbf{A}_{k}=\operatorname{diag}\left(\sigma_{0}\left(a_{k}\right), \sigma_{1}\left(a_{k}\right), \ldots, \sigma_{n-1}\left(a_{k}\right)\right)
$$

Where $a_{k} \in \mathcal{O}_{\mathbb{K}}$. In the same way, the transmitted vectors are $\mathbf{x}_{k}=\operatorname{diag}\left(\sigma_{0}\left(x_{k}\right), \sigma_{1}\left(x_{k}\right), \ldots, \sigma_{n-1}\left(x_{k}\right)\right)$, with $x_{k} \in$ $\mathcal{O}_{\mathbb{K}}$. The received vector is expressed in (1). Now we multiply (1) by the MMSE matrix B and get,

$$
\mathbf{B y}=\sum_{k=1}^{K} h_{k} \mathbf{B} \mathbf{x}_{k}+\mathbf{B} \mathbf{z}
$$

But we want to decode the equation $\sum_{k=1}^{K} a_{k} \mathbf{x}_{k}$, which means, the lattice point $\sum_{k=1}^{K} h_{k} \mathbf{B} \mathbf{x}_{k}$ through canonical embedding. So, equation (9) becomes,

$$
\mathbf{B y}=\sum_{k=1}^{K} \mathbf{A}_{k} \mathbf{x}_{k}+\tilde{\mathbf{z}}
$$

where $\tilde{\mathbf{z}}=\sum_{k=1}^{K}\left(h_{k} \mathbf{B}-\mathbf{A}_{k}\right) \mathbf{x}_{k}+\mathbf{B} \mathbf{z}$ is the equivalent noise. Minimizing the mean square error is equivalent to minimize,

$$
\begin{gathered}
\varepsilon=\frac{1}{n} \mathrm{E}\left[\tilde{\mathbf{z}}^{\dagger} \tilde{\mathbf{z}}\right] \\
=\frac{P_{x}}{n} \sum_{k=1}^{K} \operatorname{Tr}\left[\left(h_{k} \mathbf{B}-\mathbf{A}_{k}\right)\left(h_{k} \mathbf{B}-\mathbf{A}_{k}\right)^{\dagger}\right]+N_{0} \operatorname{Tr}\left[B B^{\dagger}\right]
\end{gathered}
$$

where $P_{x}$ is the per user power and $N_{0}$ is the per component noise variance. Let us now rewrite the equation (11),

$$
\begin{aligned}
& \varepsilon=\frac{P_{x}}{n} \alpha \operatorname{Tr}\left[\mathbf{B B}^{\dagger}-\mathbf{B} \mathbf{C}^{\dagger}-\mathbf{C B}^{\dagger}+\frac{1}{\alpha} \sum_{k=1}^{K} \mathbf{A}_{k} \mathbf{A}_{k}^{\dagger}\right] \\
& =\varepsilon=\frac{P_{x}}{n} \alpha \operatorname{Tr}\left[(\mathbf{B}-\mathbf{C})(\mathbf{B}-\mathbf{C})^{\dagger}+\frac{1}{\alpha} \sum_{k=1}^{K} \mathbf{A}_{k} \mathbf{A}_{k}^{\dagger}-\mathbf{C C}^{\dagger}\right] \\
& \text { with } \alpha=\|h\|^{2}+\frac{1}{\rho}, \rho=\frac{P_{x}}{N_{0}} \text { and } \\
& \qquad C=\frac{1}{\alpha} \sum_{k=1}^{K} h_{k}^{*} \mathbf{A}_{k}
\end{aligned}
$$


The minimization of $\varepsilon$ with respect to $\mathbf{B}$ is equivalent to set $\mathbf{B}=\mathbf{C}$, in which case the expression of $\varepsilon$ is

$$
\begin{aligned}
\varepsilon & =\frac{P_{x}}{n} \operatorname{Tr}\left[\sum_{k=1}^{K} \mathbf{A}_{k} \mathbf{A}_{k}^{\dagger}-\frac{1}{\alpha}\left(\sum_{k=1}^{K} h_{k}^{*} \mathbf{A}_{k}\right)\left(\sum_{k=1}^{K} h_{k} \mathbf{A}_{k}^{\dagger}\right)\right] \\
& =\frac{p_{x}}{n}\left\{\sum_{k=1}^{K} \operatorname{Tr}_{\mathbb{K} / \mathbb{F}}\left(\left|a_{k}\right|^{2}\right)-\frac{1}{\alpha} \sum_{i, j=1}^{K} h_{i} h_{j}^{*} \operatorname{Tr}_{\mathbb{K} / \mathbb{F}}\left(a_{i} a_{j}^{*}\right)\right\} .
\end{aligned}
$$

\section{B. The Compute-and-Forward With Phase Precoding}

In terms of making IA, we aim to not divide the general GS-CIC to two separate channels in parallel (create from real and imaginary parts of the original complex-valued channels). The objective is to keep the GS-CIC in its original shape, this choice will intercept of having interference between parallel channels. The same CoF protocol introduced in [9] is used in this section for GS-CIC. We propose a phase precoding scheme which is using channel coefficients as feedback to improve the results of previous works. In CoF protocol the computation rate is known as a maximal rate which users can send their codewords to a destination, this rate is given by:

$$
R(\mathbf{h}, \mathbf{a})=\frac{1}{2} \log _{2}^{+}\left\{\left(\|\mathbf{a}\|^{2}-\frac{\mathrm{SNR}\left|\mathbf{h}^{*} \mathbf{a}\right|^{2}}{1+\mathrm{SNR}\|\mathbf{h}\|^{2}}\right)^{-1}\right\}
$$

where $\mathbf{a}$ is a vector of Gaussian integers characterizing the equation we want to decode.

We are interested to improve the behavior of achievable sum-rate given by $\mathrm{CoF}$ protocol in the strong and very strong interference regimes. For 2 -user GS-CFC, $\mathbf{h}=[1, g]$ and the interference-to-noise ratio is defined as INR $\triangleq|g|^{2}$ SNR. Here, $g=\rho e^{i \phi} \in \mathbb{C}$ is the cross channel coefficient and $\mathbf{a}=$ $\left[a_{1}, a_{2}\right]$ with $a_{1}, a_{2} \in \mathbb{Z}[i]$. The bloc fading channel model is considered, then the simplified expression of (15) is

$$
R(\mathbf{h}, \mathbf{a})=\frac{1}{2} \log _{2}^{+}\left\{\frac{\left(\frac{1}{\mathrm{SNR}}+\left(1+|g|^{2}\right)\right)}{q\left(a_{1}, a_{2}\right)}\right\}
$$

where for a complex-valued channel $q\left(a_{1}, a_{2}\right)$ is a definite positive Hermitian form equal to:

$$
\begin{aligned}
q\left(a_{1}, a_{2}\right)= & \left(\left|a_{1} g\right|-\left|a_{2}\right|\right)^{2}+\frac{1}{\operatorname{SNR}}\left(\left|a_{1}\right|^{2}+\left|a_{2}\right|^{2}\right) \\
& -2 \Re\left(a_{1} a_{2}^{*} g\right)+2\left|a_{1} a_{2} g\right|
\end{aligned}
$$

We can deduce the Hermitian matrix of 17 as,

$$
G=\left(\begin{array}{cc}
|g|^{2}+\frac{1}{\mathrm{SNR}} & -g \\
-g^{*} & 1+\frac{1}{\mathrm{SNR}}
\end{array}\right)
$$

As a definite positive Hermitian form, $q\left(a_{1}, a_{2}\right)$ defines a rank 2 complex-lattice, $\Lambda_{\mathrm{CF}}$. we aim at finding the two successive minima of (17). The Hermitian form is of dimension 2 , an algorithm for finding the two successive minima is the Complex-LLL algorithm defined by [13]. This algorithm will reduce the basis of a lattice in complex field. Let define matrix $B=\operatorname{Cholesky}(G)$ be a basis of $\Lambda_{\mathrm{CF}}$ and $B_{\text {red }}$ be the reduced basis after complex-LLL reduction. $U$ is the Unimodular basis change matrix. Call $G_{\text {red }}=B_{\text {red }}^{T} B_{\text {red }}$ the reduced Gram matrix, then the two successive minima are the diagonal entries of $G_{\text {red }}$. If we fix the $\rho$ part of the cross channel coefficient, and varying its phase between $[0, \pi]$, it can be observed in Fig. 3 when the phase is approximately around 0 or $\pi$ the achievable sum-rate will be maximal. In this scenario $\arg (g)$ should be aligned to reach the maximal achievable sum-rate.

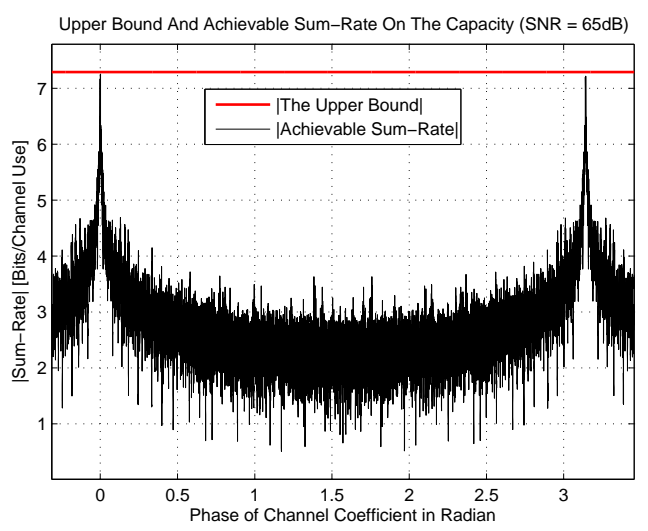

Fig. 3. Upper and lower bounds on the capacity of a 2-user GS-CIC with respect to the phase variation $\phi . g=\rho e^{i \phi}$ with $0 \leq \phi \leq \pi$ and $\rho=7.0770$ is set to be unchangeable.

After analyzing the method described in [11], we propose our precoding scheme. In our scheme each codeword should be multiplied by a precoder before transmitting them to destinations, this will let us to align their phase to maximize the final achievable sum-rate. To realize this scheme we assume that the precoder for each transmitter is a complex scalar $e^{i \theta_{k}}$, for some $-\pi / 4 \leq \theta_{k} \leq+\pi / 4$, multiplying the lattice codeword. Finding the optimal precoder factors is realized by using the CSI available as feedback information at transmitters. We apply the phase precoding function as,

$$
\begin{gathered}
D_{k}: \mathbb{C}^{n} \rightarrow \mathbb{C}^{n} \\
D_{k}\left(\mathbf{x}_{k}, \theta_{k}\right) \triangleq e^{i \theta_{k}} \mathbf{x}_{k}
\end{gathered}
$$

for $\theta_{k} \in[-\pi / 4, \pi / 4]$ and $1 \leq k \leq K$. In the $2-$ user case, we send $e^{i \theta_{1}} x_{1}$ and $e^{i \theta_{2}} x_{2}$ instead of $x_{1}$ and $x_{2}$. The phase precoded $e^{i \theta_{k}} \mathbf{x}_{k}$ continues to satisfy the power constraint (4). In general the equivalent channel coefficients vector is,

$$
\begin{gathered}
\mathbf{h}^{\prime}=\left[h_{1} e^{i \theta_{1}}, h_{2} e^{i \theta_{2}}\right] \\
=\left[\rho_{1} e^{i \times\left(\phi_{1}+\theta_{1}\right)}, \rho_{2} e^{i \times\left(\phi_{2}+\theta_{2}\right)}\right]
\end{gathered}
$$

When the channel is symmetric, $h_{1}=1$. In our proposed precoding scheme after applying precoders, the new channel coefficients are $\mathbf{h}^{\prime}=\left[e^{i\left(\theta_{1}\right)}, \rho_{2} e^{i\left(\phi_{2}+\theta_{2}\right)}\right]$, the optimal choice for precoders is therefore,

$$
\begin{aligned}
& \theta_{1}=0 \\
& \theta_{2}=-\left(\phi_{2} \pm \eta\right)
\end{aligned}
$$

$\eta \in[-0.2 \mathrm{rad},+0.2 \mathrm{rad}]$ is the precoder precision factor, it is used to keep $\left(\phi_{2}+\theta_{2}\right)$ approximately in an area which 
the rate shown in Fig. 3 is maximal. We dispose the CSI, then calculating $\theta_{2}$ is feasible by using four quadrant arctan function, $\theta_{2}=\arctan _{2}(\Im(g) / \Re(g))$. The numerical result of this scheme is presented in the next section to show its performance.

\section{Simulation Results}

The proposed scheme defined in section III was implemented with following parameters: The channel is considered as a 2 -user GS-CIC with $h_{1}=1$ and $h_{2}=\rho e^{i \phi}$. To calculate the sum-rate, SNR is chosen to be $65 \mathrm{~dB}$ and the lattice reduction is done in the complex field. For evaluating the performance of our proposed scheme and being in strong and very strong interference regimes, $\rho$ is set to vary in $\left[1.1,10^{3}\right]$ with a very high precision. Then $\phi$ is selected ramdonly in $[-\pi / 4, \pi / 4]$ for each variation of $\rho$. The reason for choosing $\phi$ in this interval, is because of using $\mathbb{Z}[i]$-lattices.

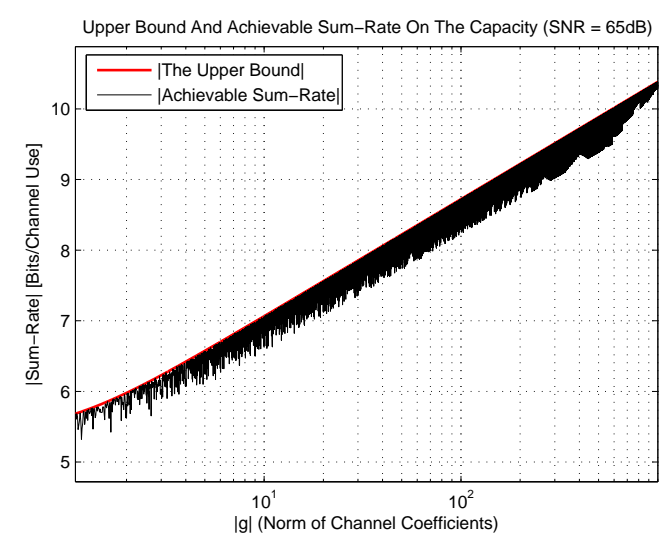

Fig. 4. Upper and lower bounds on the capacity of a 2-user GS-CIC with respect to the cross-gain. Our proposed Phase precoding technique is used.

It can be observed from Fig. 4 that for this configuration, the phase precoding scheme defined in previous section improve the achievable sum-rate of the CoF protocol [9] showed in Fig. 1 for the case of 2-user GS-CIC. As we can see in Fig. 4, the deep fadings have been limited to 0.3 [Bits/Channel Use], and there is no more gap between the upper bound and the achievable sum-rate. It means by using this scheme the interference have been aligned and also the performance of the CoF protocol has been improved.

\section{CONCLUSION}

In this paper, after studying the channel model, $\mathbb{Z}[i]$-lattice structure and the MMSE estimator matrix for the complexcase, we have showed the existence of a phase precoding scheme with partial feedback for the CoF protocol defined in [8], which will increase the finale communication rate between users. This proposed precoding scheme is enabling the relays to decode reliably to linear combination of lattice points with complex integer coefficients. Numerical results suggest that using our precoding scheme will limit the fading-like behavior and also achieve significant gain improvement in the case of 2-user GS-CIC as presented in [9]. Choosing the best precoder factor with a very high precision is essential step to improve performance of the $\mathrm{CoF}$ protocol when the interference is available. Future work will include a more detailed study of precoding schemes in the case of lattices over Eisenstein integers.

\section{REFERENCES}

[1] R. Ahlswede, "Multi-way communication channels", in Proc. IEEE International Sympos. on Inform. Theory, 2:23-52, 1974.

[2] C. E. Shannon, "Two-way communication channels," in Proc. Berkeley Sympos. on Mathematical Statistics and Probability, 1962.

[3] Syed A. Jafar, "Foundations and Trends in Communications and Inform. Theory", Vol. 7, No. 1, USA, 2011.

[4] A. S. Motahari, S. O. Gharan, M. A Maddah-Ali, and A. K. Khandani, "Real interference alignment: Exploiting the potential of single antenna systems", in IEEE Trans. Inform. Theory, 2009.

[5] A.Carlerial, "A case where interference does not reduce capacity," in IEEE Trans. on Inform. Theory, 21(5):569-570, 1975.

[6] S. Sridharan, A. Jafarian, S. Vishwanath, and S. A. Jafar,"Capacity of symmetric k-user Gaussian very strong interference channels," in Proc. IEEE Global Telecommunications Conf. GLOBECOM, pages 1-5, 2008.

[7] E. Ebrahimi Khaleghi and JC. Belfiore, "Compute-and-forward for the interference channel: Diversity precoding", in Iran Workshop on Communication and Inform. Theory, 1-6, 2014.

[8] B. Nazer and M. Gastpar, "Compute-and-forward: Harnessing interference with structured codes", in IEEE International Sympos. on Inform. Theory, pp. 772-776, 6-11 July 2008.

[9] O. Ordentlich, U. Erez and B. Nazer, "The approximate sum capacity of the symmetric Gaussian K-user interference channel", in Proceedings of the IEEE International Sympos. on Inform. Theory, pp. 2072-2076, 1-6 July 2012.

[10] N.E. Tunali, K.R. Narayanan, J. Boutros and Y.C. Huang, "Lattices over Eisenstein integers for CoF", in Annual Allerton conf. on communication, control and computing, 2012, pp. 33-40.

[11] A. Sakzad, E. Viterbo, J. Boutros and Y. Hong, "Phase Precoded Compute-and-Forward with Partial Feedback", in IEEE International Sympos. on Inform. Theory, 2117-2121, 2014.

[12] J. Conway and N. Sloane, "A fast encoding method for lattice codes and quantizers", in IEEE Trans. on Inform. Theory, vol. 29, pp. 820-824, 1983.

[13] Y.H. Gan, C. Ling and W.H. Mow, "Complex lattice reduction algorithm for low-complexity full-diversity MIMO detection", in IEEE Trans. Signal Processing, vol. 57, pp. 2701-2710, July 2009. 